\title{
Tunnel hydrodynamique destimé à l'étude de la turbulence d'écoulements eau-air
}

\author{
A hydrodynamic tunnel for water-air flow turbulence research
}

Michel Lance

Attaché de Recherche au C.N.R.S. Chargé de Recherche au C.N.R.S.

Georges Charnay
Jean Bataille*

Professeur

\section{Introduction}

Le comportement d'un milieu diphasique animé d'un mouvement turbulent et constitué d'un liquide et d'un gaz est déterminé par un grand nombre de mécanismes physiques [1]. Les effets globaux sont bien connus grâce à des travaux expérimentaux dont les plus complets portent sur des écoulements utilisés en pratique mais de structure complexe, tels des écoulements en tuyau $[2,3,4]$, des couches limites [4], des jets [5] ou des ressauts hydrauliques [6]. En fait, rares sont les études relativement simples et fondamentales consacrées à des configurations plus élémentaires et par là même plus adaptées à l'analyse systématique de chaque parametre physique, telles que celle que nous projetons dans l'installation d'essais décrite dans le présent article. Il s'agit en fait d'un tunnel hydrodynamique essentiellement conçu pour déterminer les caractéristiques turbulentes fines d'un écoulement d'eau en interaction avec des bulles d'air et aussi pour analyser le phénomène de "migration de vide".

Les. champs turbulents envisagés sont homogènes, uniformes et engendrés par une grille tandis que l'écoulement moyen, vertical, est soit uniforme soit à gradient de vitesse constant selon la configuration de la grille. La phase gazeuse est constituée par des bulles d'air introduites de façon isotherme au niveau de la grille de turbulence. Ces conditions expérimentales permettent de faire varier les parametres essentiels que sont les rapports de la dimension moyenne des bulles d'air et des diverses échelles de longueurs de la turbulence, comprises entre l'échelle intégrale liée à la maille de la grille et l'échelle de Kolmogorov. Par ailleurs, l'étude d'autres paramètres, tels que fréquence d'émission des bulles, vitesse relative

(*) Université Claude Bernard (Lyon I).

Laboratoire de Mécanique des Fluides de l'Ecole Centrale de Lyon Laboratoire Associé au C.N.R.S. des phases, propriétés physiques des fluides, peut être également abordée.

Du point de vue pratique le choix des caractéristiques géométriques et hydrodynamiques résulte d'un compromis dicté essentiellement, par des conditions sur le nombre de Reynolds, les dimensions minimales des sondes de mesure, la taille des bulles, ainsi que l'encombrement et la puissance admissibles. Les détails techniques sont fournis aux paragraphes suivants et, de façon à donner une vue plus complète des possibilités expérimentales, l'ensemble des moyens de mesure qui seront utilisés est présenté plus loin. Par ailleurs, le fonctionnement général du tunnel est précisé par les résultats des tous premiers essais de qualification. Enfin, un programme indicatif des essais futurs est proposé in fine.

\section{Dimensionnement général}

Le choix des caractéristiques d'une veine d'essais correspondant aux exigences formulées précédemment a été déterminé par les dimensions d'un réservoir existant déja au laboratoire et par les critères suivants :

- pour faciliter les comparaisons avec les travaux antérieurs, le champ turbulent doit être semblable, en particulier en ce qui concerne les nombres de Reynolds, a ceux des expériences les plus complètes en milieu monophasique,

- pour pouvoir détecter les plus petites échelles de la turbulence au moyen des sondes actuelles, les dimensions générales doivent être suffisamment grandes,

- les échelles de longueur des structures turbulentes et les tailles des bulles succeptibles d'être formées doivent pouvoir être rendues comparables.

Compte tenu de ces conditions, le dispositif expérimental retenu correspond, pour ce qui est des champs turbulents monophasiques, a l'installation de Yeh et Van 
Atta [7]. Donc les expériences réalisées avec de l'air, sont caractérisées par les grandeurs suivantes :

Dimension de la maille de la grille $\quad M=4 \mathrm{~cm}$

Echelle intégrale de longueur $\quad L \sim 2 \mathrm{~cm}$

Echelle de Kolmogorov

Nombre de Reynolds $R=U M / v \quad=10500$

Nombre de Reynolds turbulent $u^{\prime} \lambda / \nu \quad=35$

où $\nu, \lambda, U$ et $u^{\prime}$ représentent respectivement la viscosité cinématique, l'échelle de Taylor, la vitesse moyenne et la valeur efficace de la fluctuation de vitesse. Ces données ont pour avantage d'être compatibles avec les dimensions de l'ordre du millimetre des sondes de mesure et avec les tailles des bulles raisonnablement réalisables, comprises entre quelques millimètres et quelques centimètres [8]. Ces mêmes conditions expérimentales ont été conservées lors d'une étude de la turbulence thermique [7], qui pourra servir de référence lorsqu'il s'agira de très petites bulles jouant alors un rôle de marqueur passif analogue à celui de la température. Par ailleurs on retrouve les mêmes ordres de grandeur, tant pour les dimensions que pour les nombres de Reynolds dans les expériences de Champagne [9] et de Harris [10] sur des écoulements turbulents à gradient de vitesse constant.

Les dimensions de la veine d'essais sont donc déterminées en choisissant les mêmes échelles de longueur et la même valeur du nombre de Reynolds $R$ que celles de Yeh et Van Atta. Compte tenu de la substitution air-eau, elles sont données par le tableau ci-après:

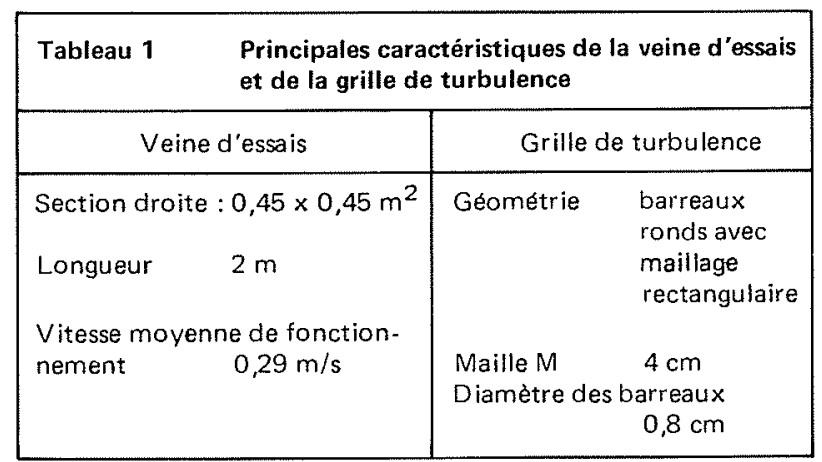

De façon à améliorer l'isotropie du champ turbulent, une contraction de rapport de sections 1,27 est intercalée entre la grille et le début de la veine d'essais [11]. Celle-ci étant située entre 11 et 66 mailles $M$ en aval de la grille on peut prévoir une intensité de turbulence, non négligeable, diminuant de $4,5 \%$ a $1,5 \%$ environ. En fin de veine, l'épaisseur des couches limites, calculée en l'absence de turbulence extérieure [12] est égale à $6 \mathrm{~cm}$, encombrement bien inférieur au coté de la veine.

\section{Descriptif détaillé}

La photo 1 donne une vue d'ensemble du tunnel dont la forme générale tient compte à la fois des caractéristiques déterminées plus haut et des contraintes d'encombrement propres au local.

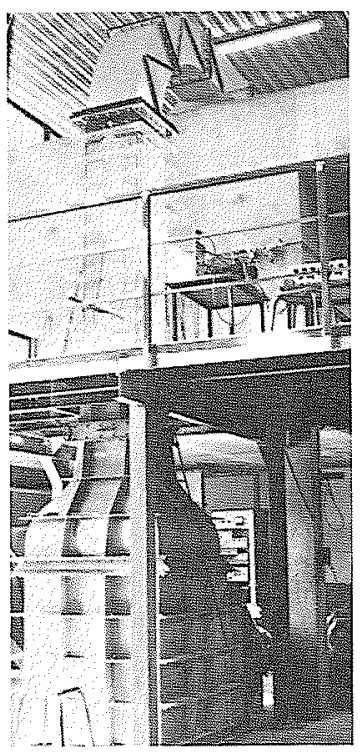

Photo 1 - Vue générale du tunnel hydrodynamique.

Principaux éléments du circuit (Fig. 1)

Dans le sens de l'écoulement on rencontre successivement :

- un bac d'aspiration $\left(4,3 \times 4,4 \times 3,1 \mathrm{~m}^{3}\right)(1)$ en ciment enduit de trois couches de peinture epoxy Sika-Dur,

- une partie (2) de tunnel démontable dans laquelle le mouvement est descendant. Selon les themes de recherche on peut y disposer, soit une veine de section droite $8 \times 40 \mathrm{~cm}^{2}$ permettant des essais acoustiques, soit la veine de $45 \times 45 \mathrm{~cm}^{2}$ spécifique à l'étude de la turbulence en milieu diphasique. Dans ce dernier cas l'écoulement et l'accélération de la pesanteur ont même sens,

- une pompe à hélice (3) entrainée par un moteur à vitesse de rotation variable,

- une vanne papillon (4),

- un ensemble constitué d'un divergent, d'un caisson de tranquilisation, d'un filtre nids d'abeille (longueur $10 \mathrm{~cm}$, maille $1 \mathrm{~cm}$ ) et d'un convergent de rapport de sections 4,6 ayant pour rôle de limiter les inhomogénéités de vitesse et la turbulence résiduelle,

- une grille (5) génératrice de turbulence et servant à injecter des bulles d'air,

- la veine d'essais principale où l'écoulement et la gravité sont de sens opposés,

- et un bac de refoulement (7) communiquant avec (1) par trois orifices de $1 \mathrm{~m}^{2}$ chacun. Les deux bacs peuvent être maintenus à niveau constant au moyen d'un déversoir.

A l'exception de la veine d'essais tous les éléments sont en acier recouvert de zinc et de peinture epoxy ou encore en bronze. Un filtrage de l'eau satisfaisant est actuellement obtenu au moyen d'un voile textile situé à l'aspiration. L'interposition de deux coudes entre la pompe et la veine d'essais (2) a pour but de diminuer les effets acoustiques du systeme moteur. 


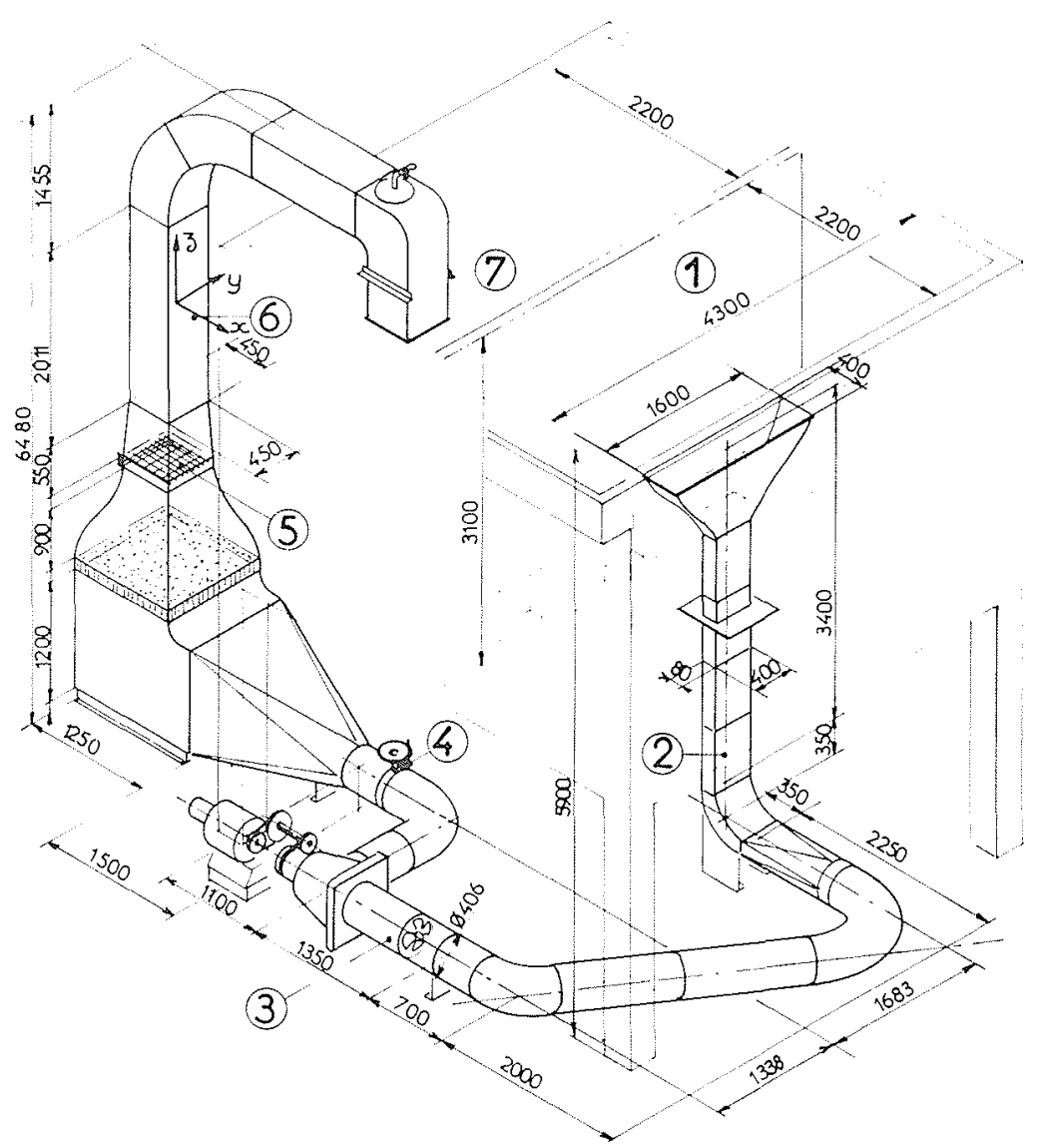

Figure 1 - Plan de l'installation.

\section{Ensemble pompe-moteur électrique}

Il s'agit d'une pompe à hélice Guinard H-400 400-4P15 fournissant un débit maximum de $0,26 \mathrm{~m}^{3} / \mathrm{sec}$ sous une charge de $2,810^{4} \mathrm{~Pa}$ et pour une vitesse de rotation de $710 \mathrm{tr} / \mathrm{min}$. Cette dernière a été volontairement limitée afin d'atténuer les bruits parasites. Néanmoins par simple changement de l'hélice et du moteur d'entraînement il serait possible de porter le débit a $0,5 \mathrm{~m}^{3} / \mathrm{sec}$.

Actuellement la pompe est entrainnée par un moteur continu dont la vitesse de rotation peut être réglée entre 100 et $2000 \mathrm{tr} / \mathrm{min}$. à $0,1 \%$ près et par une transmission de rapport de réduction égal à 2,77 .

\section{Veine d'essais (Fig. 2)}

L'ensemble de la veine est transparent, deux parois opposées étant en glace en prévision de futures visualisations ou mesures optiques et les deux autres parois étant en plexiglas de façon à obtenir une implantation commode des déplacements de sondes. Ceux ci assurent des mouvements transversaux au $1 / 10$ de $\mathrm{mm}$ et peuvent être disposés tous les $20 \mathrm{~cm}$ dans le sens longitudinal. Pour limiter les déformations, neuf serre-joints semi amovibles ont dû être rapportés.

\section{Grilles de turbulence}

Les dimensions de la grille comportant 2 plans de 12 barreaux sont données sur le tableau $n^{\circ} 1$.

L'injection en un point de l'air sous forme de bulles s'effectue à travers une conduite cylindrique parallèle à l'écoulement et soudée sur un barreau de la grille; un

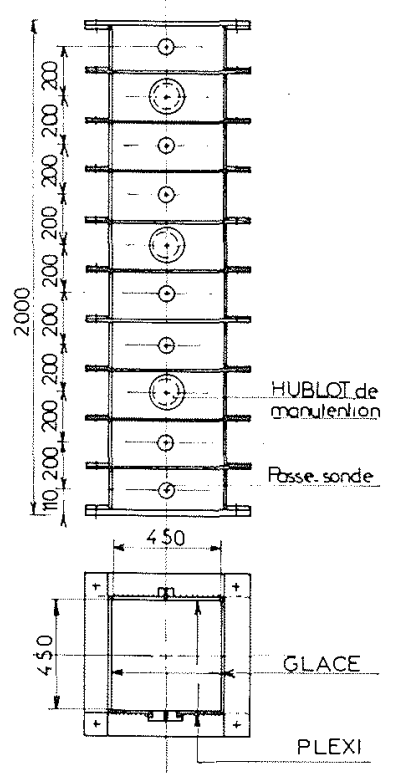


guidage des bulles peut être ainsi escompté, le choix de la longueur et du diamètre de conduite étant fixé empiriquement.

\section{Moyens de mesure}

Tout aussi importante que la construction du tunnel, l'instrumentation de la veine d'essais est en cours de développement. Les difficultés introduites par l'existence de deux phases conduit à multiplier les méthodes de détection. Le choix effectué s'appuie sur des résultats bibliographiques exhaustifs (8), (13), (14) et tient compte du matériel déjà utilisé lors d'études similaires, par exemple pour des écoulements monophasiques intermittents (15). Deux catégories de moyens expérimentaux seront mis en œuvre.

\section{Détection globale}

Un ensemble de visualisation (Matériel A.I.D. avec fonds noirs ou blancs et stroboscope etc...) constitue la pièce maîtresse de cette catégorie. Son emploi est surtout orienté vers la détermination de la trajectoire et la forme de bulles isolées et d'une façon générale vers une observation préliminaire des phénomènes.

La mesure du taux de vide moyennée sur un segment est prévue par absorption de rayonnement $\gamma$.

\section{Detection locale}

Les moyens de mesure de ce type directement utilisables sur le tunnel sont variés :

- sondes manométriques (tube de Pitot, sondes directionnelles),

- films chauds coniques (Thermosystems $n^{\circ} 1264 \mathrm{~W}$ ) ou en forme de Vé (DISA n 55 R 72) pour la mesure instantanée d'une ou de deux composantes de la vitesse,

- sonde optique (A.I.D. $n^{\circ} 7401$ ) pour la détection des phases.

Un systeme anémométrique laser à effet doppler (DISA $55 \mathrm{X}$, optique à deux couleurs) devrait aussi permettre, après une importante phase de mise au point, d'apporter des informations complémentaires.

Les signaux délivrés par ces instruments feront l'objet d'un traitement digital sur une chaine d'acquisition de données ( 8 voies, $120 \mathrm{KHz}$, précision $0,510^{-3}$, unité centrale HP 2100).

\section{Propriétés de l'écoulement}

Les résultats des tout premiers essais sont ici brièvement résumés.

\section{Observations générales}

Les essais préliminaires montrent que le réservoir permet un dégazage parfait de l'écoulement à cause de ses dimensions et de son cloisonnement en deux bacs. Par ailleurs en dépit de la construction modulaire et composite du tunnel, il n'est pas apparu de fuites, lesquelles ont pour inconvénient d'introduire des bulles parasites dans la veine.

Après la période de mise en route les dérives de température de l'eau restent limitées a 0,1 degré $C$ par 8 heures, vu le rôle régulateur du réservoir de volume total égal à $58 \mathrm{~m}^{3}$.

\section{Champ moyen}

Dans la veine d'essais la vitesse maximale de l'écoulement d'eau est $1,2 \mathrm{~m} / \mathrm{s}$.

L'uniformité du champ des vitesses moyennes dans la veine a été vérifiée, après introduction d'un grillage correcteur entre les deux convergents, avec et sans grille (Fig. 3). Dans les deux cas les écarts sont moindres que $\pm 0,7 \%$. A l'extrémité aval de la veine et avec grille de turbulence les couches limites ont un encombrement au plus égal à 0,2 fois le côté de la veine.

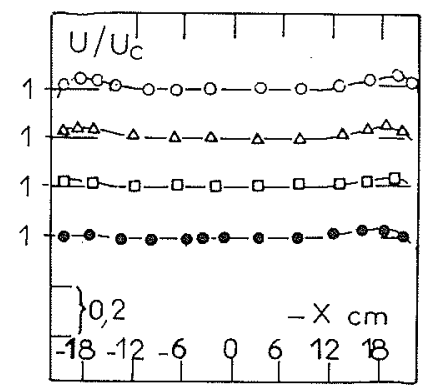

Figure 3 - Distributions transversales des vitesses moyennes. $U_{C}=U_{\text {centre }}=1 \mathrm{~m} / \mathrm{s} ;$ sans grille, $\bullet: \mathrm{y}=0 ;$ avec grille, $\square: y=+10 \mathrm{~cm}, o: y=0, \Delta=y=-10 \mathrm{~cm}$.

\section{Champ turbulent.}

L'étude de ce champ est actuellement rendue délicate par l'existence d'un phénomène de fluctuation globale à basse fréquence. En attendant d'avoir trouvé un remède satisfaisant à cet inconvénient les mesures sont obtenues en filtrant les signaux entre $2 \mathrm{~Hz}$ et $1 \mathrm{KHz}$. En l'absence de grille, la turbulence résiduelle a une intensité inférieure à $1 \%$. Le montage de la grille engendre une turbulence dont l'intensité évolue longitudinalement entre $3,5 \%$ et $1,5 \%$.

Transversalement dans la région centrale de la veine l'inhomogénéité relative d'intensité de turbulence a une valeur très satisfaisante de $1,5 \%$. 


\section{Programme expérimental envisagé.}

Par ordre chronologique les essais envisagés concernent d'abord un écoulement turbulent à champ moyen uniforme et sont relatifs a :

- l'étude de la dynamique d'une bulle isolée ou d'un train de bulles émises en un point du champ turbulent et en particulier, la détermination de l'influence de la taille des bulles,

- l'analyse de l'intéraction du champ turbulent et d'un ensemble de bulles émises en différents points. Il sera fait appel aux techniques d'échantillonnage conditionnel pour dégager les propriétés propres au seul champ turbulent.

Les mêmes étapes seront ensuite reprises dans le cas d'un écoulement de cisaillement pur.

Ce programme expérimental a évidemment pour objet une meilleure compréhension de la turbulence en écoulement diphasique ainsi que la mise en évidence des mécanismes propres à l'interaction bulles-turbulence. Par ailleurs nous pensons en tirer des indications relatives à certains termes de transfert gaz-liquide mal connus dont une modélisation convenable est essentielle pour fermer un système d'équations représentatives de l'écoulement, qui est actuellement à l'étude.

\section{Remerciements}

Les auteurs tiennent à remercier M. J.M. Delhaye, Ingénieur de recherches au Service des Transferts Thermiques du CENG, pour les conseils que lui-même et ses collaborateurs leur ont prodigués. Le concours technique de M. Dutheil a été vivement apprécié. La réalisation de cet ensemble a été rendue possible par l'aide financière accordée à ce projet par le Centre National de la Recherche Scientifique (A.T.P. 76 A 651 2809).

\section{Références bibliographiques}

[1] HEWITT G.F. et BOURE J.A. - Some recent results and development in Gas-Liquid Flow a Review. Int. J. Multiphase Flow, 1973, Vol. 1, No 1, p. 139-171.

[2] DELHAYE J.M. - Contribution à l'étude des écoulements diphasiques eau-air et eau-vapeur. Thèse de Doctorat èsSciences Physiques, Université de Grenoble, 1970.

[3] SERIZAWA A., KATAOKA I. et MICHIYOSKI I. - TUrbulence structure of air-water bubbly flow. Int. J. Multiphase Flow, 1975, vol. 2, N ${ }^{\circ} 3$, p. 221-259.

[4] HERRINGE R.A. et DAVIS M.R. - Structure development of gas-liquid mixture flows. J. Fluid Mechan., 1976, vol. 73 , part 1, p. 97-123.

[5] HETSRONI G. et SOKOLOV M. - Distribution of mass, velocity and intensity of turbulence in a two-phase turbulent jet. J. Applied Mech., 1971, 38, p. 315-327.

[6] RESCH F. - Etude expérimentale de la turbulence dans les écoulements hydrauliques. Thèse de Doctorat ès-Sciences Physiques, Université de Provence, 1974.

[7] YEH T.T. et VAN ATTA C.W. - Spectral transfer of scalar and velocity fields in heated-grid turbulence. J. Fluid Mech., 1973, vol. 58, part 2, p. 233-261.

[8] GALAUP J.P. - Contribution à l'étude des méthodes de mesure en écoulement diphasique. Thèse de Docteur Ingénieur, Université Scientifique et Médicale de Grenoble et Institut National Polytechnique de Grenoble 1975.

[9] CHAMPAGNE F.H., HARRIS V.G. et CORRSIN S. Experiments on nearly homogeneous turbulent shear flow. $J$. Fluid Mech., 1970, vol. 41, part 1, p. 81-139.

[10] HARRIS V.G. - Experimental studies in a field of nearly homogeneous sheared turbulent flow. Doctoral Dissertation, Johns Hopkins University, 1974.

[11] COMTE-BELLOT G. et CORRSIN S. - The use of a concentration to improve the isotropy of grid-generated turbulence. J. Fluid Mech., 1966, vol. 25, part 4, p. 657-682.

[12] SCHLICHTING H. - Boundary layer theory, 1968, Mc. Graw-Hill, p. 599.

[13] HEWITT G.F. - The role of experiments in two-phase systems with particular reference to measurements techniques, dans Progress in Heat and Mass Transfer, (editeurs Hestroni G., Sideman S., et Hartnett J.P., Pergamon), 1972, vol. 6, p. 295-343.

[14] DELHAYE J.M. - Measurement techniques for studies of two phase-Phase Gas-liquid Flows, dans Two-Phase Flows and Heat Transfer, (éditeurs Kakac S. et Mayinger F., Hemisphere P.C.), 1977, vol. 1, p. 37-58.

[15] SCHON J.P. et CHARNAY G. - Conditional sampling, dans Measurement of Unsteady Fluid Dynamic Phenomena (editeur Richards B.E., Hemisphere P.C.) 1977, p. 291-325. 